\title{
Ship plume dispersion rates in convective boundary layers for chemistry models
}

\author{
F. Chosson ${ }^{1, *}$, R. Paoli ${ }^{1}$, and B. Cuenot ${ }^{1}$ \\ ${ }^{1}$ URA CNRS/CERFACS no. 1875, Toulouse, France \\ *now at: Environnement Canada, Montréal, Canada
}

Received: 29 February 2008 - Published in Atmos. Chem. Phys. Discuss.: 9 April 2008

Revised: 3 July 2008 - Accepted: 3 July 2008 - Published: 21 August 2008

\begin{abstract}
Detailed ship plume simulations in various convective boundary layer situations have been performed using a Lagrangian Dispersion Model driven by a Large Eddy Simulation Model. The simulations focus on the early stage (1-2h) of plume dispersion regime and take into account the effects of plume rise on dispersion. Results are presented in an attempt to provide to atmospheric chemistry modellers a realistic description of characteristic dispersion impact on exhaust ship plume chemistry. Plume dispersion simulations are used to derive analytical dilution rate functions. Even though results exhibit striking effects of plume rise parameter on dispersion patterns, it is shown that initial buoyancy fluxes at ship stack have a minor effect on plume dilution rate. After initial high dispersion regimes a simple characteristic dilution time scale can be used to parameterize the subgrid plume dilution effect in large-scale chemistry models. The results show that this parameter is directly related to the typical turn-over time scale of the convective boundary layer.
\end{abstract}

\section{Introduction}

Studies and estimates of the impact of ship traffic on climate, atmospheric chemistry and air quality have received increasing attention in the last few years, following the observed and expected augmentation of seaborne trade, the world fleet, and the consequent increasing contribution of shipping to the world fuel consumption and anthropogenic atmospheric emissions (Eyring et al., 2005a and b; Corbett and Köhler, 2003). As repeatedly pointed out by Corbett (e.g. Corbett, 2003), ship emissions are of major concern for both researchers and politicians, and represent serious eco-

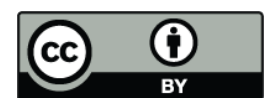

Correspondence to: R. Paoli (roberto.paoli@cerfacs.fr) nomic, environmental, technological, climate and also health (Corbett et al., 2007) challenges from local to global scales.

However, all modeling and measurement (remote or "insitu") studies of ship exhaust effects must deal with the emission process, from ship funnel to background air via inplume chemistry and background/plume mixing processes. Those entrainment processes impact plume chemistry beyond the simple exchange/dilution effects due to highly nonlinear reaction rates and can significantly alter model results at any scale (von Glasow et al., 2003; Esler et al., 2004; Poppe et al., 1998). Additionally, they can bias the interpretation of experimental measurements (Schlager et al., 2006; Chen et al., 2005; Richter et al., 2004). Supported by measurements, it has been shown that ignoring or misrepresenting chemical conversion inside the plume can lead to important overestimation of $\mathrm{NO}_{\mathrm{x}}$ and $\mathrm{O}_{3}$ emissions in global models (Esler et al., 2004; von Glasow, 2003; Davis et al., 2001). Those systematic biases especially arise from the dilution process during the early stage of plume dispersion that is before the plume has been sufficiently diluted throughout the boundary layer (Esler, 2003; Chen et al., 2005; Schlager et al., 2006; Song et al., 2003; Poppe et al., 1998).

Although such dilution effects on plume chemistry simulations have been widely considered in recent studies (e.g. Esler, 2003 and references therein), detailed descriptions of the dispersion regimes in realistic boundary layer are still lacking, and chemical modelers have to rely on parsed observations (e.g. von Glasow et al., 2003) or simple theoretical approaches using Gaussian plume models and homogeneous turbulence (Poppe et al., 1998). The present study relies on detailed and realistic plume dispersion data generated by a Lagrangian Particle Dispersion Model (LPDM) coupled to an atmospheric Large Eddy Simulation (LES) model. The objective is two-fold: first is to characterize the early stage of plume dilution in representative convective boundary layers; and second to propose a simple parameterization of dilution in chemical box and transport models.

Published by Copernicus Publications on behalf of the European Geosciences Union. 
This paper is organized as follows: the models, methodology and simulation set-up are presented in Sect. 2; in Sect. 3, we discuss the results of the simulations obtained with LPDM-LES coupled models, including the influences of boundary layer height and the initial buoyancy flux at ship stack on plume dispersion patterns; in Sect. 4, we present a methodology to derive dilution rate estimates at early stage of plume dispersion, for four different convective boundary layer situations, and various initial buoyancy flux at ship stack. The same simulations are finally used to determine a simple constant dilution rate suitable for subgrid-scale parameterization of effective emissions in coarse chemistry transport models; conclusions are given in Sect. 5.

\section{Models and methodology}

\subsection{LPDM and LES Models}

A solution to simulate realistic ship plume dispersion is to represent it by a large number of passive particle trajectories in a Lagrangian framework using time-dependent velocity fields from LES model outputs. Since the pioneering work of Lamb (1978), this method has been successfully employed in various boundary-layer and plume cases (e.g. Mason, 1992; Gopalakrishnan and Avissar, 2000; Weil et al., 2004; Cai et al., 2006).

The spatial and temporal evolution of ship plumes are simulated using DIFPAR (Wendum, 1998), a stochastic LPDM based on a Markovian "zeroth order" equation. The code has been developed by Electricité de France R and D and adapted to the scale of LES and ship emissions issue by CERFACS. The model uses periodic wind, turbulence and thermodynamic input fields which are linearly interpolated in space and time, to compute trajectories of passive particles. Those fields are provided by LES of marine boundary layers using the Non-Hydrostatic atmospheric model Meso-NH (Lafore et al., 1998), jointly developed by CNRM (Meteo-France Toulouse) and Laboratoire d'Aérologie (CNRS Toulouse). This model has been conceived to simulate air motions at all scales ranging from synoptic scale to turbulent large eddies. In the present study, Meso-NH simulations are performed in its LES mode. A complete description of the MesoNH model can be found at: http://mesonh.aero.obs-mip.fr/ mesonh. The model has been extensively used for studies of LES of various atmospheric phenomena, notably in the marine boundary layer, and especially for cloud studies (e.g. Cosma-Averseng et al., 2003; Chosson et al., 2006; Geoffroy et al., 2007).

\subsection{Plume rise scheme}

The heat release from ship exhaust stacks represents an additional buoyancy flux that controls plume dispersion, especially close to the source. In some cases, the combined effect of momentum and buoyancy may lead to plume rise of
2 to 10 times the actual release height (Arya, 1999), thus reducing the maximum ground concentration by a factor up to 100 (Briggs, 1984). In turn, the structure of the atmospheric boundary layer (ABL) can strongly modify the rise and shape of the plume as in the case of a slightly decoupled boundary layer (e.g. Liu et al., 2000). However, an exact description of the effect of buoyancy forces on particle motion is intrinsically not feasible in a Lagrangian approach because of the nature of entrainment processes that must take into account all fluid-flow interactions simultaneously (i.e. the interactions between fluid particles and between particles and background flow). Consequently, most of the Lagrangian formulations use separate plume rise models that include the effect of entrainment, by means of analytical or semi-empirical formulae of bulk plume properties, such as those derived by Briggs (1975) or similar approaches. The vertical velocity generated by these hybrid LagrangianEulerian models is then added to the Lagrangian particle motion (Luhar and Bitter, 1992; Anfossi et al., 1993; Hurley and Physick, 1993; Hurley, 1999). In our approach, the plume rise scheme added to DIFPAR model is based on the idea of Anfossi et al. (1993). The scheme is a simple parameterization that uses a generalized form of Briggs plume rise formula (Anfossi, 1985):

$H(t)=2.6\left(\frac{F t^{2} / u}{t^{2} S+4.3}\right)^{1 / 3}$

where $H$ is plume height above stack, $\bar{u}$ and $S$ are respectively the local wind module and the stability parameter, provided by the LES model; while $\bar{F}$ is the initial buoyancy flux given by:

$\bar{F}=g w_{0} r^{2} \frac{T_{f}-T_{a}}{T_{f}}$

where $w_{0}$ is the initial exhaust air velocity; $r$ is the stack radius; and $T_{f}$ and $T_{a}$ are the exit plume and ambient temperatures. Note that the estimation of $\bar{F}$ is usually complicated, due to its dependence on ship engine power and sailing configuration, background air temperature, and eventual additional exhaust devices. For diesel engine-equipped, regular cruising ocean-going vessels, estimated values of $\bar{F}$ range from 80 to $250 \mathrm{~m}^{4} \mathrm{~s}^{-3}$ with a typical mean value around $120 \mathrm{~m}^{4} \mathrm{~s}^{-3}$ (Hobbs et al., 2000; Pingkuan et al., 2006; Moldanova, 2007).

The basic idea is to assume that each particle is by itself a plume that rises according to Eq. (1) independently on the others. In order to mimic mutual particle interaction effects on buoyancy, and to fit the empirical plume radius increase near the stack (Anfossi et al., 1993), a normally distributed $F_{i}$ is assigned to each $i$ th particle such that:

$F_{i}=\bar{F}+\frac{\bar{F}}{3} \delta_{i}$ 

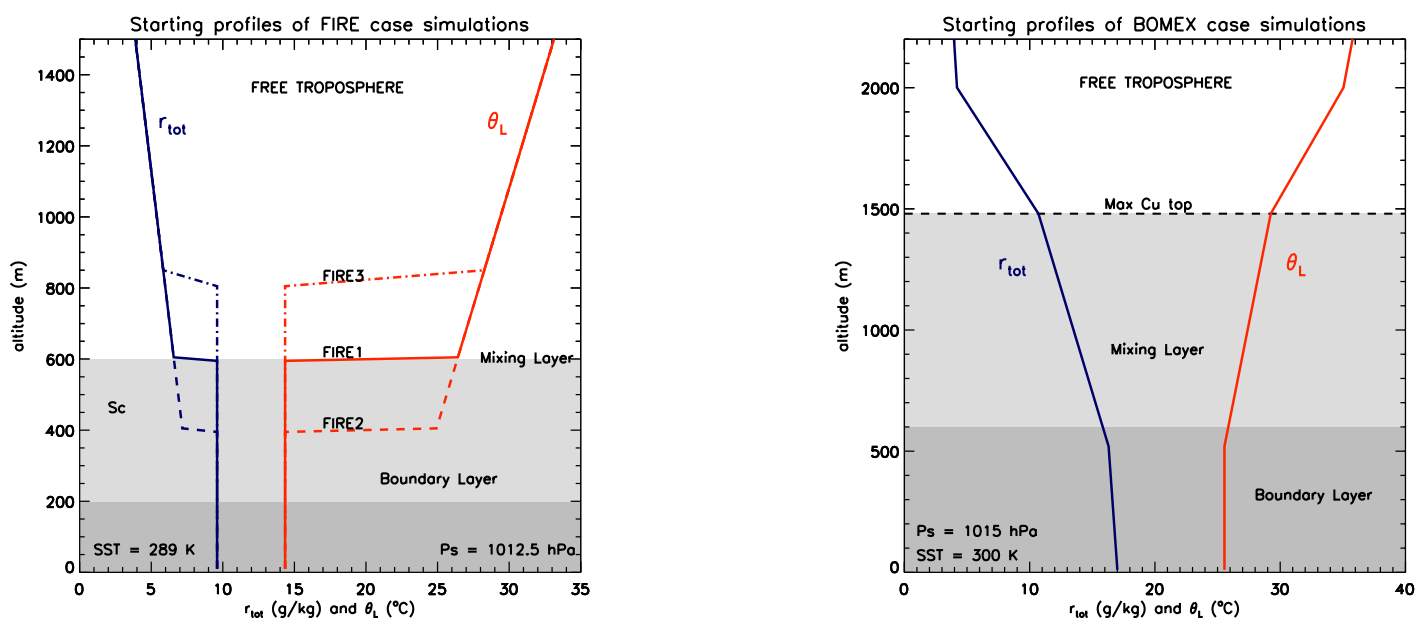

Fig. 1. Initial profiles of total mixing ratio and liquid water potential temperature for atmospheric boundary layer simulations of FIRE cases (left) and BOMEX case (right).

where $\delta_{i}$ is a Gaussian random number with zero mean and unit variance. Finally, an additional vertical velocity $w_{b}$ due to buoyancy forces is added to the particle motion at each time step:

$w_{b}=\frac{\Delta z_{b}}{\Delta t}=\frac{H(u, S, t+\Delta t)-H(u, S, t)}{\Delta t}$

The plume rise scheme is applied to each particle until $w_{b}$ is less than the local vertical turbulent fluctuations.

\subsection{Simulations set-up}

The Cloud-Topped Marine Boundary Layer observed during First ISCCP Regional Experiment (FIRE), at San Nichollas Island, 14-17 July 1987, is simulated with Meso-NH, using initial conditions provided by the EUROCS model comparison exercise (Duynkerke et al., 2004). FIRE is a well documented experiment of a typical convective marine boundary layer, and a benchmark validation test for the atmospheric modelling community. It has been successfully simulated by Meso-NH in LES mode, for validation against measurements and within intercomparison studies (Sandu et al., 2005; Sandu, 2007). The simulation has a spatial resolution of $50 \times 50 \times 10 \mathrm{~m}^{3}$ and horizontally cyclic domain size of $20 \times 5 \times 3 \mathrm{~km}^{3}$, large enough to prevent side effects and constraints on the development of convective cells. Atmospheric profiles exhibit a neutral boundary layer of about $600 \mathrm{~m}$ depth capped by a strong inversion (Fig. 1 left panel), and moderate to strong ABL wind with no shear. This simulation will be hereafter named FIRE1 case. Similarly, two other simulations named FIRE2 and FIRE3 cases, are performed with the same set-up than before, but with modified initial profiles of liquid water potential temperature $\theta_{L}$ and total water mixing ratio $r_{\text {tot }}$, in order to modify the bound- ary layer height. To that end, $\theta_{L}$ and $r_{\text {tot }}$ are kept constant in the boundary layer, but the temperature and humidity jump characterizing the inversion layer is set at the desired altitude, above which the profiles follow the initial free troposphere slope (Fig. 1 left panel). The resulting boundary layer heights are $400 \mathrm{~m}$ for FIRE2 case and $800 \mathrm{~m}$ for FIRE3 case. All FIRE simulation cases ran for one hour after $2 \mathrm{~h}$ of spinup, in order to reach a fully developed, steady, well-mixed boundary layer.

In addition, a fourth kind of boundary layer is simulated based on the convective steady state conditions observed during the Barbados Oceanographic and Meteorological Experiment (BOMEX; Holland and Rasmusson, 1973). This case served as a basis for a well known LES intercomparison study, and is fully described in Siebesma et al. (2003). In the present study we used the same domain size and resolution of FIRE cases, with a $2 \mathrm{~h}$ simulation run. The atmospheric profiles (see Fig. 1 right panel) show a $600 \mathrm{~m}$ neutral boundary layer, capped by conditionally unstable mixing layer (weak inversion). The moderate to strong wind is constant in the $\mathrm{ABL}$, with weak shear above.

Each of the four simulations can be identified by the two key parameters that directly control plume dispersion: the boundary layer depth $z_{i}$ and the turnover time scale $t^{*}=z_{i}$, with $w^{*}$ representing the convective velocity scale defined here as in Eq. (5):

$w^{*}=\left(\int_{0}^{z_{i}} g \frac{\overline{w \theta_{v}}}{\theta_{v}} d z\right)^{1 / 3}$

where $\theta_{v}$ is the horizontally averaged virtual potential temperature and $\overline{w \theta_{v}}$ is the mean vertical buoyancy flux. The 
Table 1. Boundary layer depth $z_{i}$ and characteristic turn-over time scale $t^{*}$ of the simulated BL cases.

\begin{tabular}{lcccc}
\hline & FIRE1 & FIRE2 & FIRE3 & BOMEX \\
\hline$z_{i}$ & 580 & 400 & 800 & 600 \\
$t^{*}(\min )$ & 22.2 & 12.3 & 29.2 & 23.5 \\
\hline
\end{tabular}

values of these two parameters are presented in Table 1, and are used as scaling factors to characterize plume dispersion.

\subsection{Simulating a characteristic plume}

One major issue in the simulation of ship plumes - e.g. for validation of parameterizations or comparison with observations - is that the shape of the plume strongly depends on the local flow conditions around each particle trajectory. For example, the rise and fall of a plume are influenced by extremely scattered and random events such as the updrafts and downdrafts occurring in a turbulent convective boundary layer. Besides, the exact values of the ship speed and direction (relative to the wind) are generally unknown. All these uncertainties and the wide range of spatial and temporal variability of the parameters make any single simulation of the plume a particular case among huge number of possible combinations. On the other hand, simulation of all conceivable cases is obviously unaffordable. A reasonable compromise made in this study is to reconstruct the properties of a generic ("idealized") plume that is somehow representative of any plume evolving in a given ABL. This done in two steps as detailed next.

The first step is to consider that a plume is the superposition of puffs released at different times and different locations. The above mentioned generic plume model can then be treated using one single puff simulation. The second step is to model such a puff - characteristic of the whole boundary layer - so as to get rid of the dependency on local timechanging dynamical events. The idea is to release a large number of particles equally distributed in space and eventually in time at the same ship stack level over all the modeled boundary layer.

The time evolution of the normalized concentration field of this "generic puff", characteristic of the given boundary layer, can then be recombined from the particle positions at time $t$ following (adapted from Lamb, 1978):

$$
\begin{aligned}
& C(x, y, z, t)=\int_{-\infty}^{t} Q\left(t^{\prime}\right) p_{1}\left(x_{p}(t)-x_{0}-U \times\left(t-t^{\prime}\right),\right. \\
& \left.y_{p}(t)-y_{0}-V \times\left(t-t^{\prime}\right), z_{p}(t), t^{\prime}\right) d t^{\prime}
\end{aligned}
$$

where $Q$ is the source strength function which can be time dependent; $x_{p}(t), y_{p}(t)$ and $z_{p}(t)$ indicate the particle position at time $t ; x_{0}$ and $y_{0}$ indicate the initial particle position at its release time $t^{\prime} ; U, V$ are horizontal mean wind speed components of the ABL in the case of a stationary source. In the case of a moving source, $(U, V)$ can be interpreted as the relative wind speed components $\left(U_{\text {wind }}-U_{\text {ship }}, V_{\text {wind }}-V_{\text {ship }}\right)$. $p_{1}$ is the position PDF for particles found at time $t$ in a coordinate system that depends on the particle initial position and the mean advection. The method is thus particularly suitable for practical implementation in atmospheric chemistry models which rely only on general boundary layer parameters. It must however be noted that Eq. (6) accurately describes any generic puff provided that the statistic properties of turbulence is independent enough from the mean wind velocity, which is the case in convective boundary layers but not for shear-driven boundary layers.

In our cases, the LES fields are stored every minute, provided off-line to the Lagrangian model and interpolated in time and space (taking advantage of the cyclic boundary conditions of the LES domain); 50000 particles are released instantaneously, equally distributed horizontally over the domain, at an initial altitude of $60 \mathrm{~m}$, matching the upper limit of ship height above sea level in international PANAMAX standard for major marine vessels (which defines the maximum size allowed for the crossing of the Panama Canal, and corresponds to the design of modern container ships).

\section{Analysis of plume dispersion parameters}

\subsection{Influence of ship stack buoyancy flux on plume disper- sion}

The mean initial buoyancy flux at ship stack $\bar{F}$ (Eq. 2) is a tunable parameter for the puff dispersion simulation. The plume rise scheme is applied to each particle following Eq. (3). For each boundary layer case, seven plume simulations are performed with a mean initial buoyancy flux $\bar{F}=0 \mathrm{~m}^{4} \mathrm{~s}^{-3}$ (no buoyancy), 50, 100, 120, 150, 200 and $250 \mathrm{~m}^{4} \mathrm{~s}^{-3}$.

Although the initial buoyancy flux can have a major impact on plume dispersion as discussed above, in situ observations show that the heat and humidity release at ship stack can not significantly raise the in-plume temperature and water mixing ratio above background level, even as close as $200 \mathrm{~m}$ from the stack. In rare and specific cases, only a small temperature increase of less than $0.4^{\circ} \mathrm{K}$ has been documented (Hobbs et al., 2000).

In the simulations, the temperature difference between plume and boundary layer background can be estimated using the plume rise scheme integrated in the Lagrangian model. For each particle and at each time step, a vertical buoyancy acceleration is obtained by taking time derivative of its plume rise velocity $w_{b}(t)$ defined in Eq. (4). This acceleration can be related to temperature difference between 
the plume air particle and the surrounding environment via a buoyancy force:

$$
\frac{\partial w_{b}}{\partial t}=\frac{\Delta w_{b}}{\Delta t} \approx g \frac{\theta_{v}-\theta_{v} \text { ref }}{\theta_{v} \text { ref }}
$$

where $\theta_{v}$ ref is the virtual potential temperature of environment, provided by the LES fields. However, simulation results show that the heat flux at ship stack has little impact on the estimated plume temperature, which drops quickly to background value. This is reported in Fig. 2 that reports the maximum temperature excess with respect to background as a function of time and altitude for a initial buoyancy flux at ship stack of $250 \mathrm{~m}^{4} \mathrm{~s}^{-3}$; almost twice our estimated mean value for a cruising large ocean-going vessel (Moldanova, 2007). This result is in agreement with in-situ measurements of temperature close to the ship stack.

Although the buoyancy force is active only during early stage (a few minutes) of puff evolution, it indirectly affects the dispersion of the plume on time scales of the order of several convective turnover times of the boundary layer. This is shown in Fig. 3 that reports the evolution of the normalized mean vertical concentration, defined as in Cai et al. (2006) by

$C_{\text {vert }}(z, t)=z_{i} \int_{X Y} \int_{\text {plane }} C(x, y, z, t) d x d y$

for various initial buoyancy fluxes in the BOMEX case. In this case, the high sensitivity of the simulations to this parameter can be explained by the fact that the boundary layer is capped by a weakly stable inversion layer. Thus, if particles get sufficient buoyancy or are driven by strong convective burst, they can overshoot the boundary, and eventually stay trapped in the upper layer (Liu et al., 2000), as clearly shown in Fig. 3 for $\bar{F}=250 \mathrm{~m}^{4} \mathrm{~s}^{-3}$. This possibility is prevented in the FIRE simulations due to the strong capping inversion, so that all particles remain inside the boundary layer. However, the initial buoyancy flux has again a strong impact on the plume dilution pattern. In fact, up to three convective turnover times are needed to reach a final state independent of $\bar{F}$ (not shown). Note that differences are not significant for values larger than $100 \mathrm{~m}^{4} \mathrm{~s}^{-3}$.

\subsection{Influence of boundary layer height and turnover time scale on plume dispersion}

The height of the boundary layer has two effects on the evolution of the plume and its shape. The first (direct) effect is that it sets a global length scale for dilution and defines different regimes of dilution in the case of convective boundary layers. As shown in Fig. 4, a typical exhaust buoyant puff first disperses quickly into the boundary layer until its upper portion reaches the inversion height. As a result, the dilution process then slows down. On the other hand, the lower portion of the puff falls down and touches the ground soon after, which again reduces the dilution rate of the mean puff concentration. Note that there are situations, depending on the

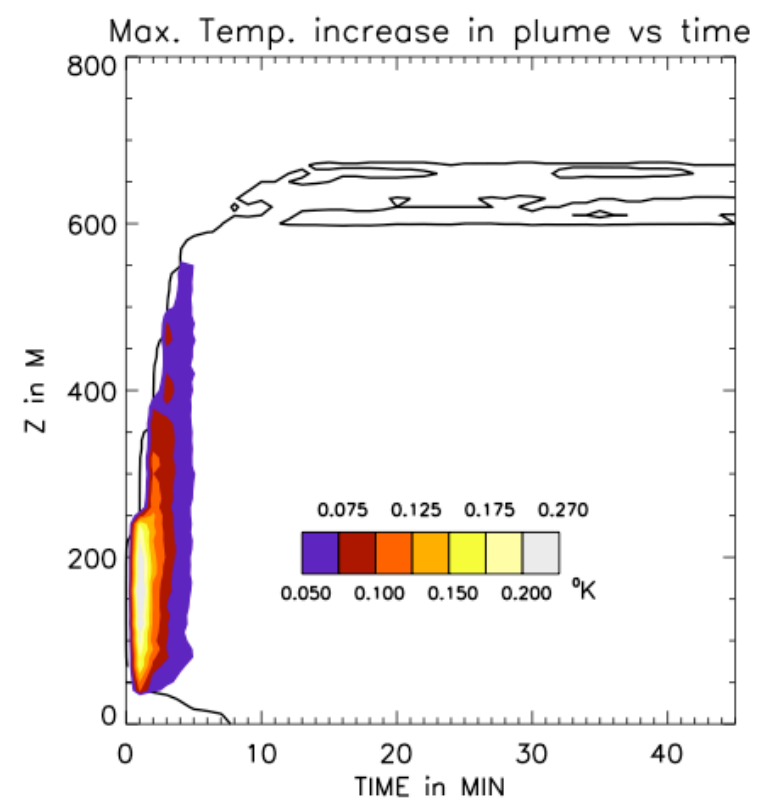

Fig. 2. FIRE1 case: maximum temperature difference excess with respect to background for an initial buoyancy flux $\bar{F}=250 \mathrm{~m}^{4} \mathrm{~s}^{-3}$. The black line represents the plume envelope (including all particles).

initial buoyancy flux, the height of exhaust release and the inversion height, where the first two dilution regimes cannot be clearly separated. Once the puff is roughly well mixed in the vertical direction, its dilution is mainly driven by horizontal dispersion.

The second (indirect) effect of boundary layer depth is that the inversion height controls the size and turnover time of convective cells and then impacts the dispersion process of the plume. Figure 5 shows the normalized mean vertical concentration for the FIRE1, FIRE2, FIRE3 and BOMEX cases. In the FIRE2 case, with $t^{*} \approx 12 \mathrm{~min}$, the puff dispersion in the vertical direction is driven by the boundary layer convective pulsation. This feature is also present in the BOMEX case, although the inversion is less permeable (see the discussion in previous section). This pattern is not clearly seen in the FIRE1 case where $t^{*} \approx 22 \mathrm{~min}$, i.e. only one third of the total simulation run-time, and much larger than the onset of the third dilution regime. That is to say that dilution is too fast compared to larger-scale turbulent convection, whose action is outweighed by small-scale dispersion. (Note that, for the same reason, in the FIRE3 case there are no initial "waving" patterns). For all simulated cases, the second indirect effect becomes negligible after a few convective turnover time scales, when the plume can be considered as "well mixed". 

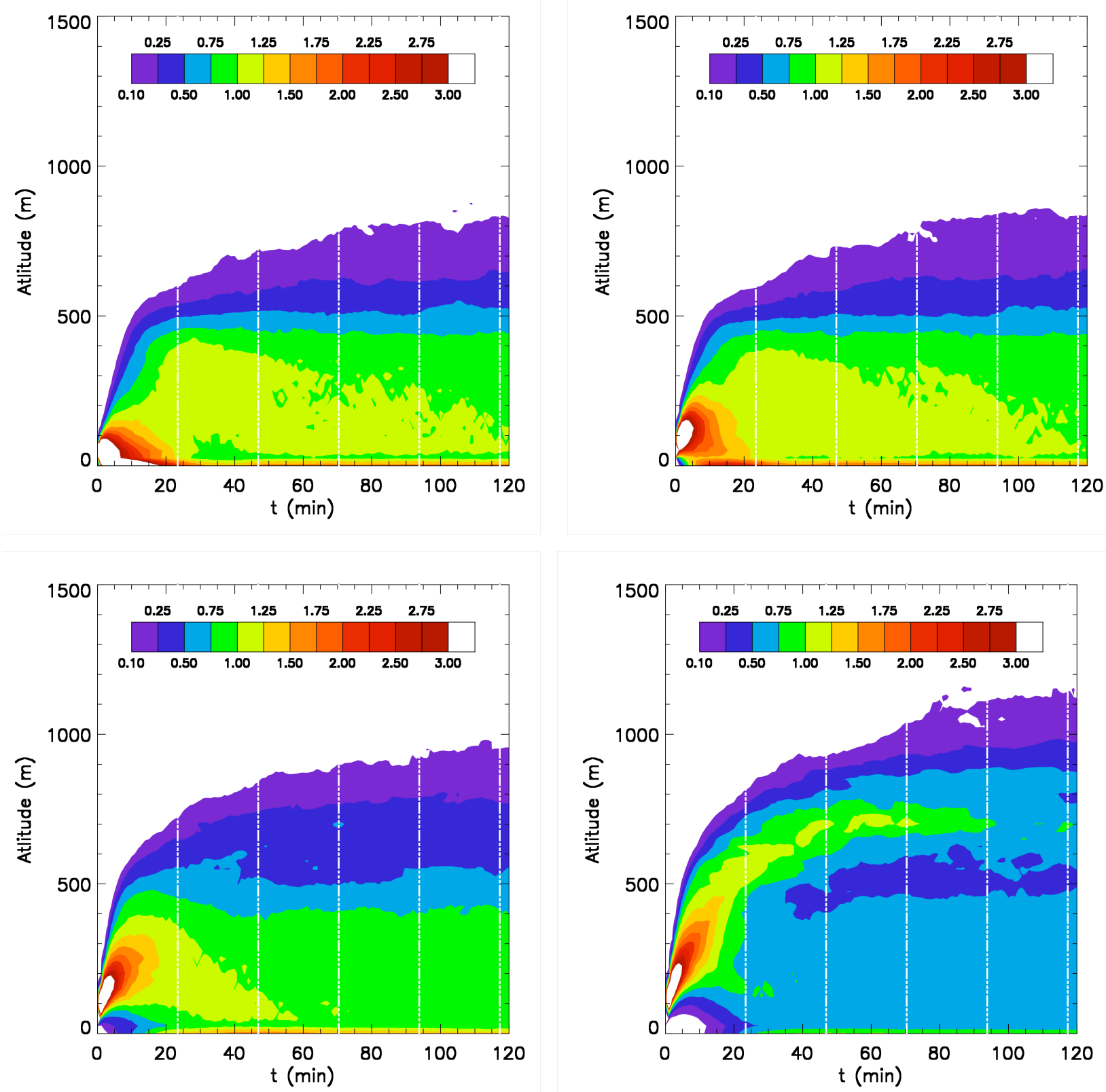

Fig. 3. Time evolution of the normalized mean vertical concentration for BOMEX case, and an initial mean buoyancy flux $\bar{F}=0 \mathrm{~m}^{4} \mathrm{~s}^{-3}$ (top left), $50 \mathrm{~m}^{4} \mathrm{~s}^{-3}$ (top right), $120 \mathrm{~m}^{4} \mathrm{~s}^{-3}$ (bottom left) and $250 \mathrm{~m}^{4} \mathrm{~s}^{-3}$ (bottom right). The vertical dashed-dotted lines represent the characteristic turnover time scale of the boundary layer. 

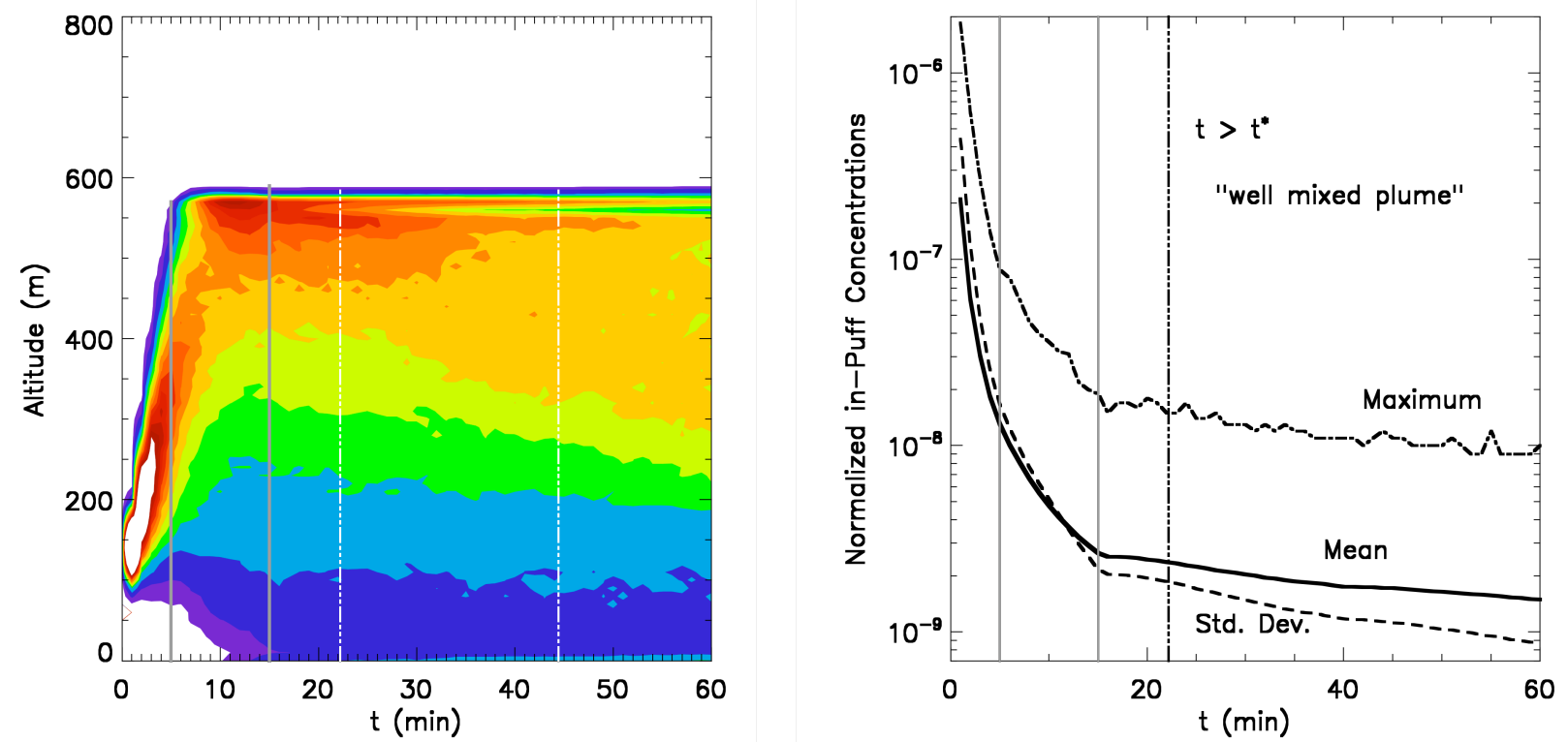

Fig. 4. FIRE1 case: example of the 3 dilution regimes of the puff (see text), for an initial buoyancy flux $\bar{F}=250 \mathrm{~m}^{4} \mathrm{~s}^{-3}$. Left: time evolution of normalized vertical mean concentration. Right: time evolution in logarithmic scale of the mean, maximum and standard deviation of inpuff normalized concentration. The vertical continuous grey lines separate the three dilution regimes. The vertical dot-dashed lines represent the convective turnover time scale $t^{*}$.

\section{Estimate of the plume dilution rate}

In chemical box models (that use an explicit plume model; see e.g. von Glasow et al., 2003) or in chemistry transport models (where the plume is parameterized at subgrid scale level), mixing is in generally represented using two air masses where chemical species are assumed to be homogeneously distributed: the well mixed background (e.g. the boundary layer) and the plume itself. The dilution process and the way it influences the plume chemistry is then controlled by the exchange rate between these two air masses via an entrainment/mixing law:

$$
\left.\frac{\partial \overline{C_{p}}}{\partial t}\right|_{\operatorname{mix}}=-\left(\overline{C_{p}}-\overline{C_{a}}\right) D(t)
$$

where $\overline{C_{p}}$ is the mean in-plume concentration of a given chemical species (or aerosol species); $\overline{C_{a}}$ is the mean homogeneous background concentration, while $D(t)$ is the (unknown) dilution rate to be determined.

\subsection{Dilution rate for chemical box models}

In our model, the plume is considered as a superposition of puffs, and mixing occurs only at edge of the puff via entrainment of background air in the crosswind plane (see e.g. von Glasow et al., 2003). Thus, we neglect the puff dilution in the downwind direction (the so-called "slender plume approximation"), which is fully justified in our simulations since wind is not nil and we do not consider dispersion very close to the source. The plume cross-wind surface concentration field, at any time $t$ after release (age of the plume), can thus be derived from the generic puff 3-D concentration field $C(x, y, z, t)$ by simple integration along the downwind direction $x: C_{\text {plume }}(y, z, t)=\int C(x, y, z, t) d x$. In this case the change in concentration within the plume is related to the change in puff surface $A_{p}$ on the cross-wind plane such that:

$D(t)=\frac{1}{A_{p}} \frac{d A_{p}(t)}{d t}=\frac{d \ln \left(A_{p}\right)}{d t}$

Three different definitions of the puff surface have been examined depending on the representation of the puff/background interface: the first one consists of the surface that contains $98 \%$ of the emitted particles; the second one takes the core of the plume that contains $60 \%$ of the particles; and the last one defines the surface as:

$A_{p}(t)=A \sigma_{y} \sigma_{z}(t) \propto \sigma_{y}(t) \sigma_{z}(t)$

where $\sigma_{y}$ and $\sigma_{z}$ are the dispersion parameters in crosswind and vertical directions, respectively (in practice, the standard deviations of puff concentrations, see Niewstadt, 1992; Dosio et al., 2003). However, we did not find significant differences in the results (not shown) using these three definitions, thus we retained the last one, Eq. (11) as it provides smooth variation of plume area, and is more easily related to the widely used Gaussian plume models in the literature. Figure 6 presents the natural logarithm of the plume crosswind surface for all simulations as a function of non-dimensional 

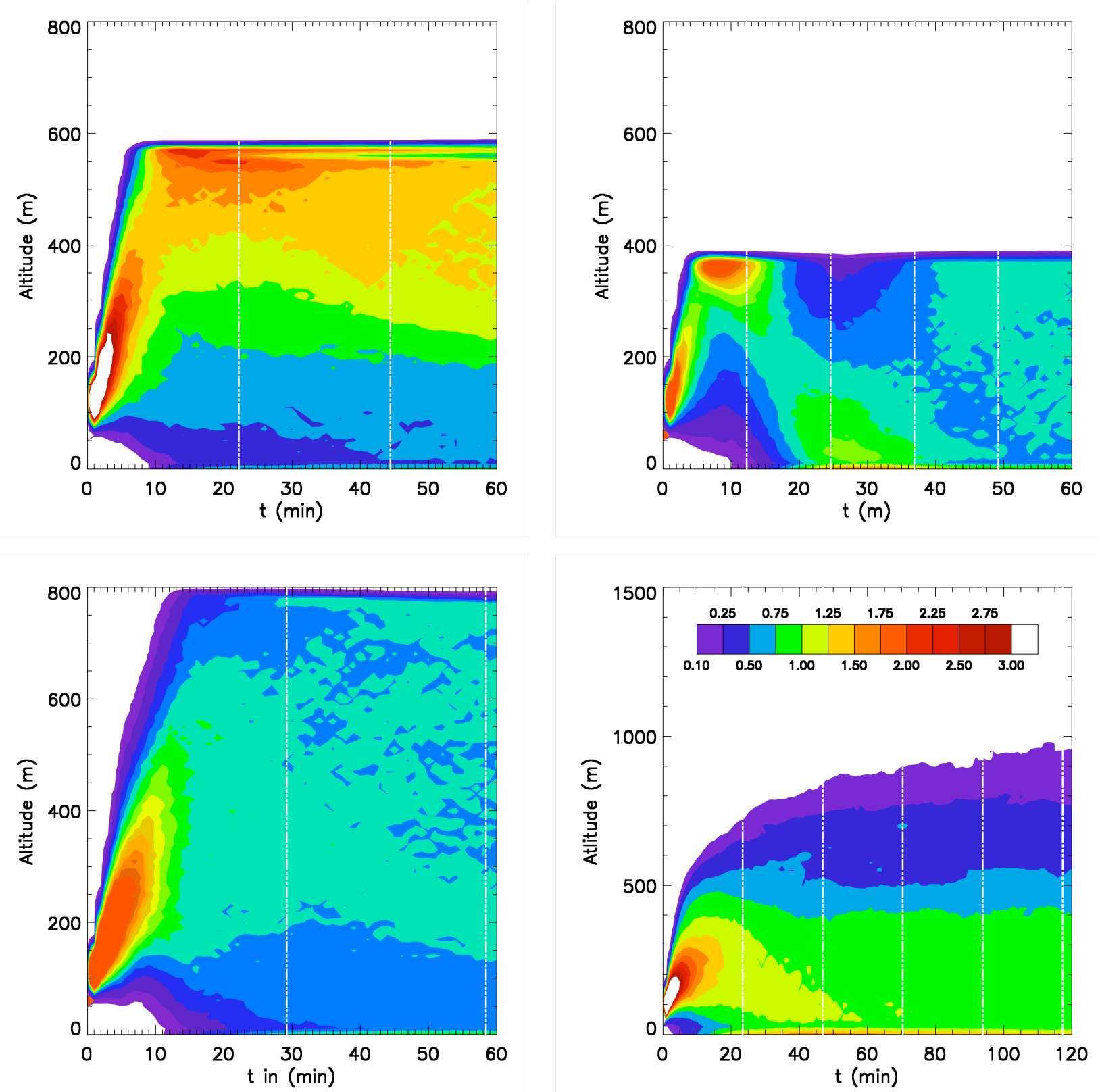

Fig. 5. Time evolution of normalized mean vertical concentration for FIRE1 (top left), FIRE2 (top right), FIRE3 (bottom left) and BOMEX (bottom right) with initial mean buoyancy flux $\bar{F}=120 \mathrm{~m}^{4} \mathrm{~s}^{-3}$. The vertical dashed-dotted lines represent the characteristic turnover time scale of each boundary layer. 


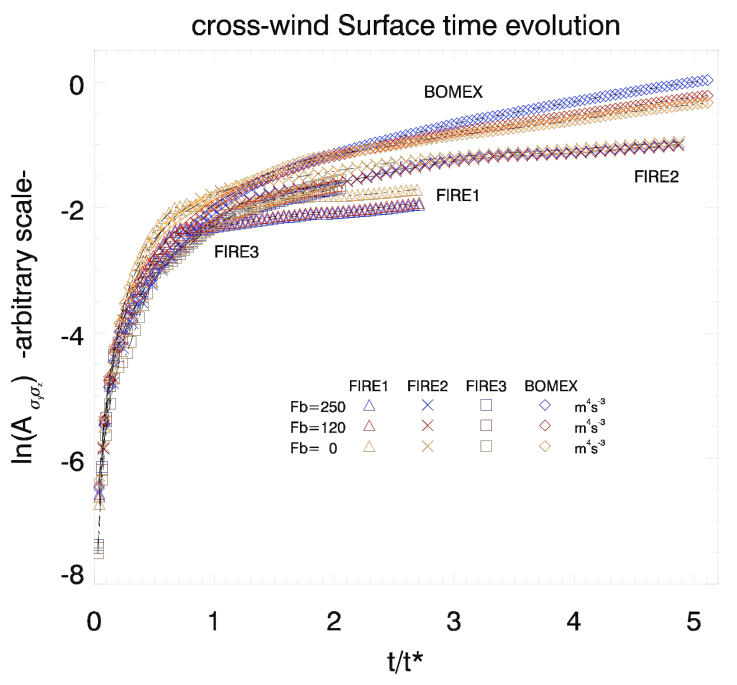

Fig. 6. Logarithm of the crosswind plume surface (arbitrary unit) as a function of non-dimensional time for all four boundary layer cases, and initial buoyancy flux $\bar{F}=0$ (no plume rise), 120 and $250 \mathrm{~m}^{4} \mathrm{~s}^{-3}$.

Table 2. Best fit results for $D(t)=a\left(t^{*} / t\right)^{b}$ considering all simulations results, or as a function of mean initial buoyancy flux $\bar{F}=0$, 120 , or $250 \mathrm{~m}^{4} \mathrm{~s}^{-3}$. Also shown are goodness of fit parameters $S y . x$ (standard deviation of the residuals) and $R^{2}$.

\begin{tabular}{lcccc}
\hline Simulation Cases & $a\left(\mathrm{~min}^{-1}\right)$ & $b$ & $S y . x\left(\mathrm{~min}^{-1}\right)$ & $R^{2}$ \\
\hline $\bar{F}=0 \mathrm{~m}^{4} \mathrm{~s}^{-3}$ & 0.043 & 1.12 & $7.94 \times 10^{-3}$ & 0.79 \\
$\bar{F}=120 \mathrm{~m}^{4} \mathrm{~s}^{-3}$ & 0.049 & 1.11 & $6.37 \times 10^{-3}$ & 0.82 \\
$\bar{F}=250 \mathrm{~m}^{4} \mathrm{~s}^{-3}$ & 0.051 & 1.08 & $5.37 \times 10^{-3}$ & 0.84 \\
All cases & 0.046 & 1.07 & $5.46 \times 10^{-3}$ & 0.84 \\
\hline
\end{tabular}

time $t / t^{*}$. This scaling eases comparisons among boundary layers with their own convective time scale $t^{*}$, which is a common parameter characterizing the convective ABL in generic dispersion studies (see e.g. Fedorovich, 2004). The dilution rate function $D(t)$ is obtained from crosswind surface data using Eq. (10) and is presented in Fig. 7 for all simulations. The figure suggests that dispersion in the boundary layer is characterized by the competition between the buoyant plume dilution regimes and convection processes, as discussed above. This results in abrupt changes in the derived instantaneous dilution rates that is clearly visible in the logarithmic scale, especially for FIRE1 case (note that data in Fig. 6 were not smoothed out for the derivation). Meanwhile note that the computed dilution rates were found to be almost insensitive to the initial buoyancy flux, despite the rather scattered nature of the plume vertical dispersion patterns shown in the previous section.

One of the goals of this paper is to provide the characteristic dilution rate for convective BL that can be used in a simple

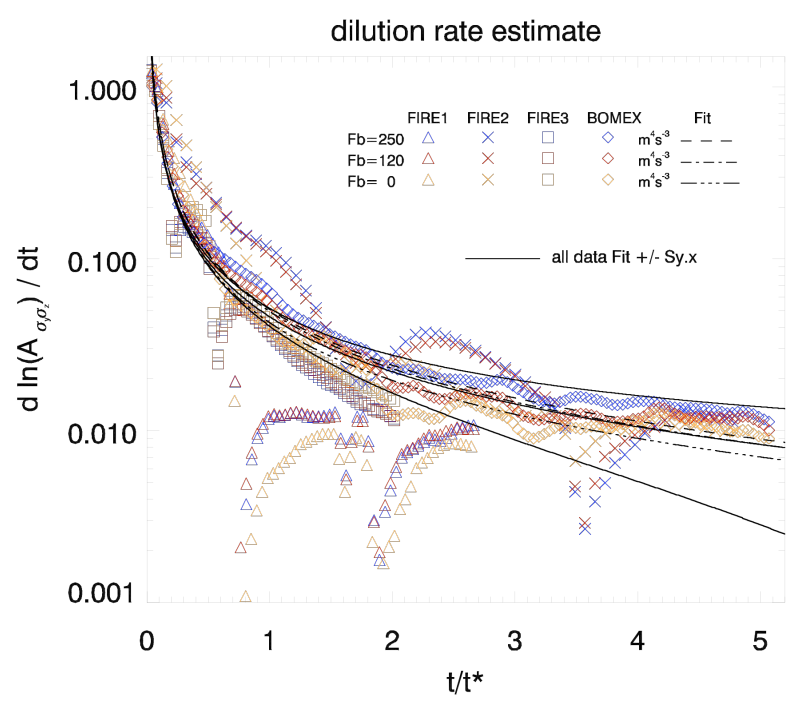

Fig. 7. Dilution rate estimate: logarithmic derivative of plume crosswind surface as a function of non-dimensional time, for all boundary layer cases and initial buoyancy flux $\bar{F}=0 \mathrm{~m}^{4} \mathrm{~s}^{-3}, 120$ and $250 \mathrm{~m}^{4} \mathrm{~s}^{-3}$. Also shown are best fits for each initial buoyancy fluxes cases (dashed and dot-dashed lines), and all data (solid lines). Note that results are presented in logarithmic scale.

and straightforward way into chemistry transport models. To that end, we propose a power-law function that best fits the simulation results:

$D(t)=a\left(\frac{t^{*}}{t}\right)^{b}$.

Table 2 reports the values of the fitting parameters $a$ and $b$, while Fig. 7 presents the evolution of best-fitted dilution function rate in Eq. (1), together with the curves extracted from the simulations for various initial buoyancy fluxes. Note that derived dilution rates $D(t)$ are in minutes. Both Table 2 and Fig. 7 confirm the minor effect of initial buoyancy flux on plume dilution rate estimates. It is also interesting to note that the results of Table 2, with best fits values $b$ and $a t^{*}$ both close to unity, suggest that isotropic two-dimensional Fickian diffusion (which leads to a Gaussian plume model) within the slender plume approximation, represents a good description of ship plume dilution in convective boundary layers.

\subsection{Dilution rate for chemical transport model}

In chemical plume models, such those included in mesoscale, regional or global circulation models, the plume is usually parameterized as a subgrid-scale process. Indeed, a detailed representation of the plume dispersion would require grid resolutions that are not affordable by present supercomputers. One must find out a more simple description of the dilution rate function $D(t)$ that does not change within a characteristic time smaller than the time step of such models 
(or simply independent on time). Moreover, in those models, the plume is not considered as a superimposition of puffs, but rather a single puff which diffuses slowly within the grid cell.

We propose to look for a plume dilution rate in a given boundary layer in the form

$D(t)=\frac{1}{\tau}$

which is similar in spirit to the exchange-with-the-mean models (Dopazo and O'Brien, 1974) that are often used in other areas of turbulence research such as in turbulent combustion to represent subgrid-scale mixing (see, e.g. Ren and Pope, 2004 for a review). In Eq. (13), the characteristic dilution time scale $\tau$ is found by fitting the mean in-puff particle concentration derived from a single puff simulation, for each initial buoyancy flux. This dilution rate should not depend on the initial setup of the puff diffusion and the early stage of the dispersion when buoyancy is still dominant and the puff has not yet reached a steady evolution (i.e. the first two dilution regimes described in Sect. 3). The fit is then made for $t>t^{*}$

To do so, we linearly fit the logarithm of the normalized mean in-puff particle concentration evolution starting at a time equal to the convective turnover time $t^{*}$. This is justified from the linear behaviour of this mean concentration, as seen in the right panel of Fig. 4. The absolute value of the line slope thus gives the characteristic dilution rate $1 / \tau$. Figure 8 presents the characteristic dilution time $\tau$ scaled by $t^{*}$ as a function of the initial mean buoyancy flux, for all simulation cases.

The figure indicates the same evolution for all boundary layers situations: initially $\tau$ decreases as $\bar{F}$ increases, and quickly converges to a constant value for $\bar{F}>100 \mathrm{~m}^{4} \mathrm{~s}^{-3}$. This is due to the fact that initial buoyancy flux acts as an accelerator of plume dilution until it reaches a "well-mixed" state throughout the boundary layer, that is when dilution relies mainly upon convective related processes. In other words, for small $\bar{F}$, this state can be not fully reached within a period $t^{*}$, as discussed in Sect. 3. This clearly shows the importance of using more sophisticated methods to represent dilution in boundary layers, compared to standard Gaussian plume models, where plume rise is either totally neglected or taken into account by simply lifting the initial emission source.

Figure 8 also shows that, for initial buoyancy fluxes of typical ocean-cruising vessels values ( $\bar{F}=80$ to $\left.250 \mathrm{~m}^{4} \mathrm{~s}^{-3}\right)$, all scaled dilution times exhibit similar values of about $\tau / t^{*} \approx 4.12 \pm 0.47$. This interesting result suggests a general (far-field) dispersion law since the data shown in Fig. 8 come from very different boundary layers (especially BOMEX versus FIRE cases), and very different plume dilution patterns. Furthermore, the range of ship stack buoyancy flux considered in this study also suggests that vessel type or size has a minor impact on plume dispersion. Nevertheless, this has to be confirmed by additional simulations and observations (for example using the simulation results and tank experiments

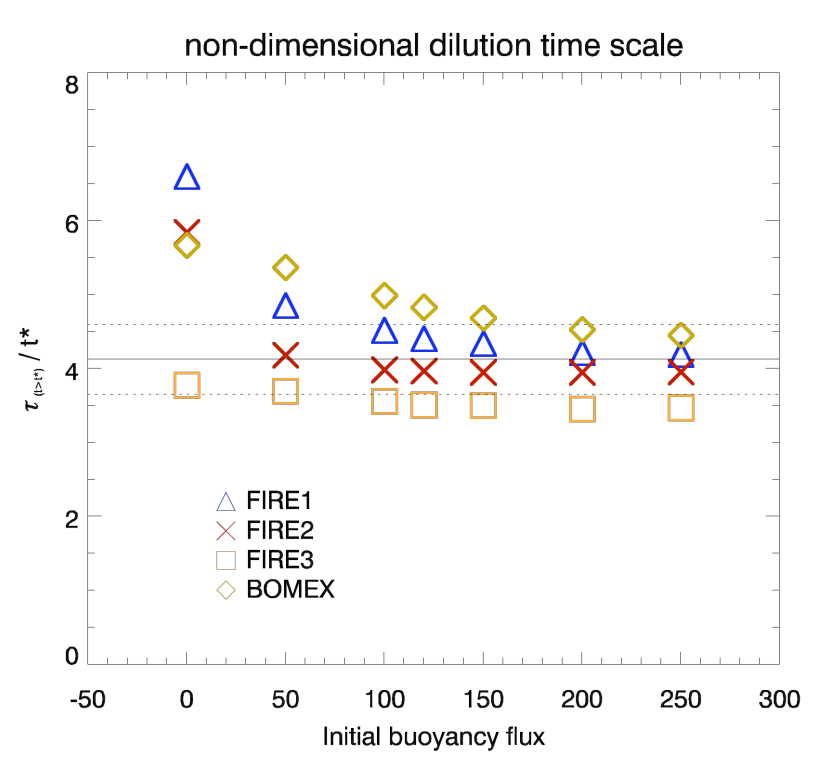

Fig. 8. Dilution rate estimate: best fit results of non-dimensional constant dilution time scale estimate for third dilution regime, as a function of initial buoyancy flux for all boundary layer cases. Also shown are mean (solid line) \pm standard deviation (dotted lines) for initial buoyancy fluxes corresponding to estimated typical ocean-going vessels values ( $\bar{F} \approx 80$ to $250 \mathrm{~m}^{4} \mathrm{~s}^{-3}$ ).

reported in Fedorovitch 2004), and/or by theoretical work to derive a more systematic parameterization.

The result is however encouraging, as it provides a simple parameterization of dilution in chemical transport models. Indeed, the boundary layer height $z_{i}$, the surface convective velocity $w^{*}$, and then $t^{*}$, are directly available from standard outputs of most atmospheric models. Note that this is only justified in convective boundary layer, which is a common situation over ocean surfaces.

The proposed parameterization should be extended to cover the initial dilution regime (the dilution time scale is effectively constant only after the plume gets mixed with background). In such a parameterization, one should take into account dispersion processes from the source to this steady dilution regime, using, for example:

(i) the detailed results of dispersion presented in the previous section (but the computational gain of a parameterization would be lost);

(ii) instantaneous release of ship exhaust injected directly at a well-mixed stage throughout the BL (but biases are expected in net chemical products);

(iii) equivalent or effective emissions (Esler, 2003; Paoli et al., 2008 ${ }^{1}$; Cariolle, 2007) that account for dispersion

\footnotetext{
${ }^{1}$ Paoli, R., Cariolle, D., Cuenot, B., Paugam, R., and Chosson, F.: Modeling chemical reactions and emissions from concentrated sources into global models, C. R. Mechanique, submitted, 2008.
} 
from the source to the diluted stage (additional CPU cost is then only limited by prior off-line computations).

In this context, and despite its simplicity, the dilution model defined by Eq. (13) (or other models without explicit time dependence) would certainly be easier to implement in global chemistry models than the "exact" diffusion Eq. (12). Such a choice remains however an important open modelling issue that deserves further investigation.

\section{Summary and conclusion}

The present paper was motivated by the increasing concern about the environmental impact of ship traffic emissions. Although it is now recognized that the early stage of plume dispersion has a strong impact on plume chemistry, chemistry models generally rely on rough parameterizations of plume dispersion or simple Gaussian plume models.

The aim of this study was to provide a realistic description of the entrainment-mixing processes between plume and background air that can be easily incorporated into chemical box models or large scale chemistry transport models. To that end, detailed ship plume simulations were performed within various convective boundary layers, using a Lagrangian Particle Dispersion Model (LPDM) driven by high resolution atmospheric Large-Eddy Simulations (LES). The analysis focused on early stage of plume dispersion, i.e. the first onetwo hours after release. The LPDM contains a plume rise scheme to improve the plume representation and explore the impact of the initial buoyancy flux at ship exhaust funnel on plume dispersion.

The different plume dilution regimes up to the well-mixed plume steady state were clearly identified in the simulations. The impact of the plume rise scheme, boundary layer height and characteristic convective turnover time scale on plume dispersion was also discussed. Analytical dilution rate functions were derived as they are classically used in chemical box models. These rates were found to depend on the convective turnover time scale of the boundary layer, but are almost insensitive to the initial buoyancy flux, despite its striking effect on plume dispersion patterns.

Finally, a subgrid plume dispersion model for chemistry transport models was proposed. The plume dispersion was parameterized by a constant dilution rate characteristic of the well-mixed dilution regime. This parameter was found to be weakly dependent on the plume rise scheme for low values of the initial buoyancy flux, but not for values encountered in typical ocean-going vessels. Interestingly, the dilution time scaled with the characteristic turnover time of the convective boundary layer by a factor of about four in all simulations considered in this study.

These results are promising for the simplicity of the model and the computational ease of implementation in large scale chemistry transport models. However, despite the number of boundary layers and plume cases analyzed in this study, more effort is still needed to derive a general parameterization, especially for shear-driven convective boundary layers. This is left for future work.

Acknowledgements. We thank the reviewers for their helpful suggestions. This present study was supported under grants from QUANTIFY, Integrated Project funded by the European Commission and national funding agencies (http://www.pa.op.dlr.de/quantify/). We are grateful to EDF R\&D for the possibility to use DIFPAR model code.

Edited by: J. Seinfeld

\section{References}

Anfossi, D., Ferrero, E., Brusasca, G., Marzorati, A., and Tinarelli, G.: A simple way of computing buoyant plume rise in lagrangian stochastic dispersion models, Atmos. Environ., 27(A), 9, 14431451, 1993.

Anfossi, D.: Analysis of plume rise data from five TVA steam plants. J. Clim. Appl. Met., 24, 1225-1236, 1985.

Arya, S. P.: Air pollution, meteorology and dispersion, Oxford University Press, New York, USA, 320 pp., 1999.

Briggs, G. A.: Plume rise and buoyancy effects, in: Atmospheric science and power production, Oak Ridge, TN, edited by: Randerson, D., US Department of Energy, Technical Information Center, 327-366, 1984.

Briggs, G. A.: Plume rise predictions, in: Lectures on air pollution and environmental Impact Analysis, American Meteorological Society, Boston, USA, 59-111, 1975.

Cai, X., Zhang, R., and Li, Y.: A large-eddy simulation and lagrangian stochastic study of heavy particle dispersion in the convective boundary layer, Bound.-Layer Meteor., 120(3), 413-435, 2006.

Cariolle, D.: Parameterization into large scale models of the nonlinearities of the atmospheric chemistry during plume dispersion, Technical Report TR/AE/07/24, CERFACS, Toulouse, France, 2007.

Chen, G., Huey, L. G., Trainer, M., Nicks, D., Corbett, J., Ryerson, T., Parrish, D., Neuman, J. A., Nowak, J., Tanner, D., Holloway, J., Brock, C., Crawford, J., Olson, J. R., Sullivan, A., Weber, R., Schauffler, S., Donnelly, S., Atlas, E., Roberts, J., Flocke, F., Hübler, G., and Fehsenfeld, F.: An investigation of the chemistry of ship emission plumes during ITCT 2002, J. Geophys. Res., 110, D10S905, doi:10.1029/2004JD005236, 2005.

Chosson, F., Brenguier, J.-L., and Schüller, L.: Entrainment-mixing and radiative transfer simulation in boundary layer clouds, J. Atmos. Sci., 64, 2670-2682, 2007.

Corbett, J. J. and Köhler, H. W.: Updated emissions from ocean shipping, J. Geophys. Res., 108(D20), 4650, doi:10.1029/2003JD003751, 2003.

Corbett, J. J., Winebrake, J. J., Green, E., Kasibhatla, P., Eyring, V., and Lauer, A.: Mortality from ship emissions: a global assessment, Environ. Sci. Technol., 41, 8512-8518, 2007.

Corbett, J. J.: New Directions: designing ship emissions and impacts research to inform both science and policy, Atmos. Environ., 37, 4719-4721, 2003. 
Cosma-Averseng, S., Flamant, C., Pelon, J., Palm, S. P., and Schwemmer, G. K.: The cloudy atmospheric boundary layer over the subtropical South Atlantic Ocean: airborne-spaceborne lidar observations and numerical simulation, J. Geophys. Res., 108(D7), 4220, doi:10.1029/2002JD002368, 2003.

Davis, D. D., Grodzinsky, G., Kasibhatla, P., Crawford, J., Chen, G., Liu, S., Bandy, A., Thornton, D., Guan, H., and Sandholm, S.: Impact of ship emissions on marine boundary layer $\mathrm{NO}_{\mathrm{x}}$ and $\mathrm{SO}_{2}$ distributions over the Pacific basin, Geophys. Res. Lett., 28, 235-238, 2001.

Dosio, A., Vilà-Guereau de Arellano, J., Holtslag, A. A. M., and Builtjes, P. J. H.: Dispersion of a passive tracer in buoyancy and shear-driven boundary layers, J. Appl. Meteorol., 42, 11161130, 2003.

Dopazo, C. and O'Brien, E. : An approach to autoignition of a turbulent mixture, Acta Astronautica, 1, 1239-1266, 1974.

Duynkerke, P. G., De Roode, S. R., Van Zanten, M. C., Calvo, J., Cuxart, J., Cheinet, S., Chlond, A., Grenier, H., Jonker, P. J., Köhler, M., Lenderink, G., Lewellen, D., Lappen, C.-L., Lock, A. P., Moeng, C.-H., Müller, F., Olmeda, D., Piriou, J.M., Sanchez, E., and Sednev, I.: Observations and simulations of the diurnal cycle of the EUROCS stratocumulus case, Q. J. R. Meteorol. Soc., 604, 3269-3296, 2004.

Esler, J. G., Roelofs, G. J., Kölher, M. O., and O’Connor, F. M.: A quantitative analysis of grid-related systematic errors in exidising capacity and ozone production rates in chemistry transport models, Atmos. Chem. Phys., 4, 1781-1795, 2004, http://www.atmos-chem-phys.net/4/1781/2004/.

Esler, J. G.: An integrated approach to mixing sensitivities in tropospheric chemistry: a basis for the parameterization of subgridscale emissions for chemistry transport models, J. Geophys. Res., 108(D20), 4632, doi:10.1029/2003JD003627, 2003.

Eyring, V., Köhler, H. W., Lauer, A ., and Lemper, B.: Emissions from international shipping - 2: Impact of future technologies on scenarios until 2050, J. Geophys. Res., 110, D17306, doi:10.1029/2004JD005620, 2005b.

Eyring, V., Köhler, H.W., van Aardenne, J., and Lauer, A.: Emissions from international shipping - 1: The last 50 years, J. Geophys. Res., 110, D17305, doi:10.1029/2004JD005619, 2005a.

Fedorovich, E.: Dispersion of passive tracer in the atmospheric convective boundary layer with wind shears: a review of laboratory and numerical model studies, Meteorol. Atmos. Phys., 87, 3-21, 2004.

Geoffroy, O., Brenguier, J.-L., and Sandu, I.: Relationship between drizzle rate, liquid water path and droplet concentration at the scale of a stratocumulus cloud system, Atmos. Chem. Phys. Discuss., 8, 3921-3959, 2008,

http://www.atmos-chem-phys-discuss.net/8/3921/2008/.

Gopalakrishnan, S. G., and Avissar, R.: An LES study of the impacts of land surface heterogeneity on dispersion in the convective boundary layer, J. Atmos. Sci., 57, 352-371, 2000.

Hobbs, P. V., Garrett, T. J., Ferek, R. J., Strader, S. R., Hegg, A., Frick, G. M., Hoppel, A., Gasparovic, R. F., Russell, L. M., Johnson, D. W., O’Dowd, C., Durkee, P. A., Nielsen, K. E., and Innis, G.: Emissions from Ships with respect to their effects on clouds, J. Atmos. Sci., 57, 2570-2590, 2000.

Holland, J. Z. and Rasmusson, E. M.: Measurement of atmospheric mass, energy, and momentum budgets over a 500-kilometer square of tropical ocean, Mon. Weather Rev., 101, 44-55, 1973.
Hurley, P. J. and Physick, W.: Lagrangian particle modeling of buoyant point sources: plume rise and entrapment under convective conditions, Atmos. Environ., 27, 1579-1584, 1993.

Hurley, P. J.: The air pollution model (TAPM) version 1: technical description and examples, CSIRO Atmospheric Research Technical Paper No. 55, 43 pp., 1999.

Lafore, J.-P., Stein, J., Bougeault, P., Ducrocq, V., Duron, J., Fischer, C., Héreil, P., Mascart, P., Masson, V., Pinty, J.-P., Redelsberger, J. L., Richard, E., and Vilà-Guerau de Arellano, J.: The Meso-NH atmospheric simulation system - Part 1: Adiabatic formulation and control simulations, Ann. Geophys., 16, 90-109, 1998, http://www.ann-geophys.net/16/90/1998/.

Lamb, R. G.: A numerical simulation of dispersion from an elevated point source in the convective planetary boundary layer, Atmos. Environ., 12, 1297-1304, 1978.

Liu, Q., Kogan, Y. L., Lilly, D. K., Johnson, D. W., Innis, G. E., Durkee, P. A., and Nielsen, K. E.: Modeling of Ship effluent transport and its sensitivity to boundary layer structure, J. Atmos. Sci., 57, 2779-2791, 2000.

Luhar, A. K. and Britter, R. E.: Random-walk modeling of buoyantplume dispersion in the convective boundary layer, Atmos. Environ., 26, 1283-1298, 1992.

Mason, P. J.: Large-eddy simulation of dispersion in convective boundary layers with wind shear, Atmos. Environ., 26A, 15611571, 1992.

Moldanova, J.: Main campaign quicklooks and preliminary data from the ship exhaust measurements, QUANTIFY European Integrated Project, internal report no. D.2.3.2.10, 2007.

Nieuwstadt, F. T. M.: A Large-Eddy simulation of a line source in a convective atmospheric boundary layer -1 : Dispersion characteristics, Atmos. Envir., 26A, 485-495, 1992.

Pier Siebesma, A., Bretherton, C. S., Brown, A., Chlond, A., Cuxart, J., Duynkerke, P. G., Jiang, H., Khairoutdinov, M., Lewellen, D., Moeng, C.-H., Sanchez, E., Stevens, B., and Stevens, D. E.: A Large Eddy Simulation Intercomparison Study of Shallow Cumulus Convection, J. Atmos. Sci., 60, 10, 12011219, 2003.

Pingkuan, D., Servin, A., Rosenkranz, K., and Schwarh, B.: Diesel particulate matter exposure assessment study for the ports of Los Angeles and Long Beach, California Environment Protection Agency, Air Ressources Board (ARB), Sacramento, CA, USA, 74 pp., 2006.

Poppe, D., Koppmann, R., and Rudolph, J.: Ozone formation in biomass burning plumes: Influence of atmospheric chemistry, Geophys. Res. Lett., 25, 3823-3826, 1998.

Ren, Z. and Pope, S. B. : An investigation of the performance of turbulent mixing models, Comb. Flame, 136, 208-216, 2004.

Richter, A., Eyring, V., Burrows, J.P., Bovensmann, H., Lauer, A., Sierk, B., and Crutzen, P. J.: Satellite measurements of $\mathrm{NO}_{2}$ from international shipping emissions, Geophys. Res. Lett., 31(23), L23110, doi:10.1029/2004GL020822, 2004.

Sandu, I., Tulet, P., and Brenguier, J.-L.: Parameterization of the cloud droplet single scattering albedo based on aerosol chemical composition for LES modeling of boundary layer clouds, Geophys. Res. Lett., 32, L19814, doi:10.1029/2005GL023994, 2005.

Sandu, I.: Impact des aérosols sur le cycle de vie des nuages de couche limite, PhD Thesis, Météo-France, Université Toulouse 
III, France, 2007.

Schlager, H., Baumann, R., Lichtenstern, M., Petzold, A., Arnold, F., Speidel, M., Gurk, C., and Fischer, H.: Aircraft-based trace gas measurements in a primary European ship corridor, in: Proceedings of the International Conference on Transport, Atmosphere and Climate (TAC), 26-29 June 2006, Oxford, UK, 8388, 2006.

von Glasow, R., Lawrence, M. G., Sander, R., and Crutzen, P. J.: Modeling the chemical effects of ship exhaust in the cloud-free marine boundary layer, Atmos. Chem. Phys., 3, 233-250, 2003, http://www.atmos-chem-phys.net/3/233/2003/.
Weil, J. C., Sullivan, P. P., and Moeng, C.-H.: The use of large-eddy simulations in Lagrangian particle dispersion models, J. Atmos. Sci., 61, 2877-2887, 2004.

Wendum, D.: Three long range transport models compared to the ETEX experiment: a performance study, Atmos. Env., 32, 42974305, 1998. 